\title{
Visual Cognitive Function Measurement System for Unilateral Spatial Neglect Using Smartphone VR
}

\author{
Yuuki Araikawa \\ Osaka Prefecture University \\ Japan \\ sya01009@edu.osakafu-u.ac.jp
}

\author{
Masashi Sugano \\ Osaka Prefecture University \\ Japan \\ sugano@kis.osakafu-u.ac.jp \\ Yasuo Naitoh \\ Osaka Prefecture University \\ Japan \\ naitoh@rehab.osakafu-u.ac.jp
}

\author{
Shinichi Takabatake \\ Osaka Prefecture University \\ Japan \\ takabata@rehab.osakafu-u.ac.jp
}

\begin{abstract}
As a method for evaluating the unilateral spatial neglect that occurs as a sequela of cerebral infarction, a system combining a display with a touch panel and a line-of-sight tracking device has been proposed. However, movement of the head cannot be avoided, and the measurement accuracy of the line of sight is not sufficient. Therefore, in this research, we propose an application of smartphonebased virtual reality as a tool for evaluating unilateral spatial neglect. Because it is realized as an application on a smartphone, it can be easily used at low cost, and it can be expected to be used in day clinics and in at-home medical care. In this study, we evaluated the system for patients and therapists by actually using the proposed system.
\end{abstract}

\section{CCS CONCEPTS}

- Applied computing $\rightarrow$ Health care information systems • Human-centered computing $\rightarrow$ Mobile phones

\section{KEYWORDS}

unilateral spatial neglect, virtual reality, smartphone, rehabilitation

\section{INTRODUCTION}

Unilateral spatial neglect (USN) is a condition in which it becomes impossible to recognize or react to stimuli on the side opposite one cerebral hemisphere $[1,2]$. USN occurs in many cases when cerebral infarction and cerebral hemorrhage arise in a cerebral hemisphere. Most often, left unilateral spatial neglect arises after right hemisphere damage. Those with USN cannot notice the fact that they are ignoring the hemisphere space. Therefore, it is reported that even during meals, people with USN cannot notice the left side of a tray placed in front of them, and will finish the meal without being able to put hands on a dish on the left side of the tray. Traditionally, evaluation by Catherine Bergego Scale (CBS) [3] based on a direct observation of the patient's functioning in 10 real-life situations, such as grooming, dressing, or wheelchair driving has been conducted. However, since there is a difference for each evaluator, it is considered that its quantitative objectivity is not sufficient.

Systems have been proposed in which PCs provide intervention measures for objective assessment and symptomatic improvement against attention disorders, including unilateral spatial neglect [4]. The system is equipped with a touch panel and consists of a display and a line of sight detection / input sensor, it is possible to record reaction time required for selecting each object displayed on the display and eye movement trajectory during execution of the task. In addition, by grasping the behavioral characteristics of both touching with the fingertip and eye movement, it is possible to obtain clues about the mechanism of inference responsible for symptoms. Furthermore, objective/quantitative evaluation of negligible symptoms and attention disorders is possible by analysis of recorded data.

However, in the conventional evaluation method, there is the problem that the line of sight cannot be accurately measured by tracking movement of the head. Attempting to solve this problem by the use of virtual reality (VR) has become popular in recent years [5]. However, a VR system that requires a high-performance PC and a dedicated head-mounted display (e.g., HTC Vive) cannot be easily used at home for the purpose of rehabilitation. In this study, we focus on VR technology using smartphones and 
propose a tool for evaluating visual cognitive function in unilateral spatial neglect.

\section{PROPOSED SYSTEM}

\subsection{Promotion of eye movement to the left}

This application presents targets with numbers on the VR space in order at a constant speed, and the subject answers with the numbers written on the targets. This makes it possible to quantify the perceptible range for subjects. Targets are presented within 60 degrees of viewing angle left and right at regular intervals. As shown in Fig. 1, it is possible to select whether to display in a straight line or in a zigzag manner within a width in the vertical direction. In addition, it is possible to set the presentation time of the target, the number of targets, and whether to keep the target displayed or delete it after a certain time. In addition, it is possible to select whether to display in both visual fields or display with only the left or right visual field.

(a) Linear target display

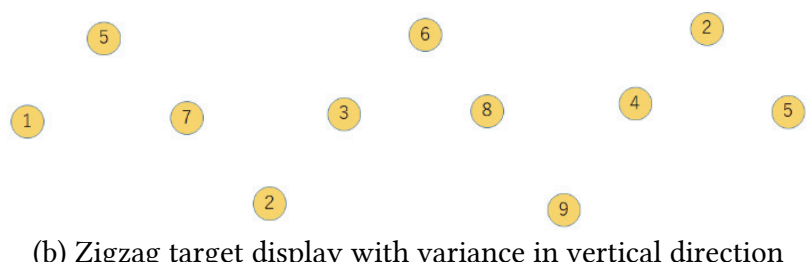

Figure 1: Promotion of eye movement to the left.

\subsection{Promotion of right-and-left eye movement}

As shown in Fig. 2, this application presents the target at a constant speed from above to below the field of view and displays the target to the left and right at the same height. The spacing of the target is widened as it is displayed lower in the view field. By allowing subjects to answer with numbers in response to the target, it is possible to quantify the perceivable range.
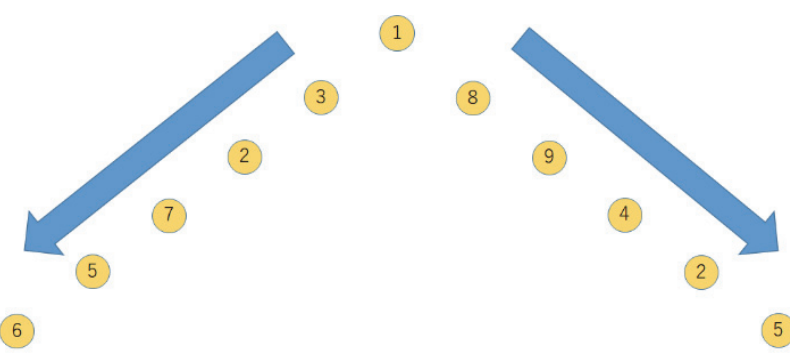

Figure 2: Promotion of right-to-left eye movement.

\subsection{Operation of proposed system}

The measurement implementer installs the developed Android application on the smartphone and starts it. At application startup, it is possible to select the presentation time of the target, the number of targets, and the display method of the target. Also, when the smartphone is attached to a head-mounted display (HMD), the measurement executor cannot see the screen, so Visor [6] is installed on smartphone, which then duplicates the screen to a PC connected via USB and enables operation from the PC.

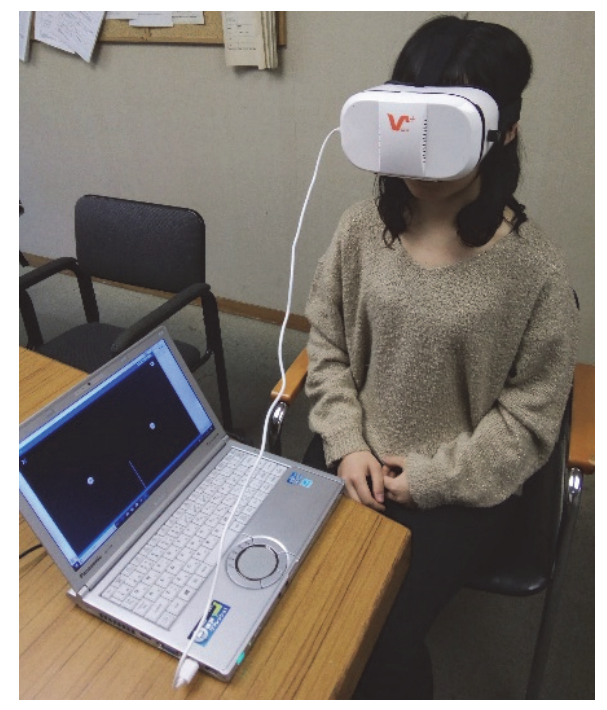

Figure 3: By installing Visor on the smartphone, the measurement executor can mirror the screen being shown on the smartphone worn by the subject on the display of the PC and can also operate the smartphone.

\section{EVALUATION BY QUESTIONNAIRE}

We asked patients and their therapists at a day service station for rehabilitation to actually use the proposed system, after which we conducted a questionnaire evaluation $(n=18)$. Questions were rated on a Likert-type scale from 5 (strongly agree) to 1 (strongly disagree). On one hand, as shown in Table 1, the questionnaire results for the therapists are high for all questions. In particular, the necessity of a tool to digitally measure the visual perception range, utility of the proposed system, and ease of operation were all high, and it is possible to ascertain a high demand for a system like the one proposed. Furthermore, free descriptions given in response to a question about the scope of application of the proposed system are shown below.

- Pediatric patients with cerebral palsy with visual perception problems 
- Patients with limited range of motion due to neck injury

- Diplopia by strabismus

- Patients with learning difficulties and those on the autism spectrum

- Use as a training tool instead of evaluation

On the other hand, the evaluation by subjects was not very high. The viewability of the image received a particularly low score, and some subjects experienced nausea due to seeing the image, and the average value of responses is lower. One reason for this that the image cannot be viewed properly on an HMD equipped with a smartphone because the range of focus adjustment is narrow. In addition, as an essential problem of VR, some sickness due to the difference with the real world may be inevitable. Others pointed out that it is difficult to see the target and it is not easy to use the HMD with glasses on.

\section{Table 1: Evaluation results from questionnaire about the proposed system (mean values)}

\begin{tabular}{ll}
\hline Evaluation by therapist & \\
\hline Necessity to measure cognitive range with digital method & 4.6 \\
\hline Utility of the proposed system & 4.6 \\
\hline Easy to understand operation & 4.6 \\
\hline Convenience of the proposed system (transportability) & 4.4 \\
\hline Evaluation by subject & \\
\hline Discomfort from mounting of HMD & 4.1 \\
\hline Visibility of images & 3.0 \\
\hline Feeling of sickness from looking at images & 3.1 \\
\hline
\end{tabular}

\section{CONCLUSIONS}

In this study, we proposed a system to evaluate visualfield recognition ability in people with semi-lateral spatial neglect, using VR on a smartphone. The proposed system does not require any special equipment and can be used easily at different sites, including for home rehabilitation. As a result of actual use, it was suggested that the potential demand was high because therapists rated the system highly. However, it became clear that there is room for improvement in the display of VR images. As a future task, we will also explore possibilities for using the tool for training as well as assessment of vision.

\section{ACKNOWLEDGMENTS}

We thank the users and therapists of the rehabilitation day service of Kanaeru Link Co., Ltd., who cooperated in the evaluation of the proposed system.

\section{REFERENCES}

[1] K. M. Heilman, R. T. Watson, and E. Valenstein. 1993. Neglect and related disorders. Clinical neuropsychology 3, 279-336.

[2] G. Vallar. 2001. Extrapersonal visual unilateral spatial neglect and its neuroanatomy. Neuroimage 14, 1 (2001), S52-S58.

[3] P. Azouvi. 2010. Functional Consequences and Awareness of Unilateral Neglect: Study of an Evaluation Scale. Neuropsychological Rehabilitation 6, 2 (2010), 133-150. DOI: 10.1080/713755501

[4] N. Kawashima, A. Kamoshida, M. Nakagawa, Y. Nozuki, and M. Yamamoto. 2015. Development of the evaluation tool for symptoms with unilateral spatial neglect. 251-257. DOI: 10.11477/mf.1552200172

[5] T. Ogourtsova, W. Souza Silva, P. S. Archambault, and A. Lamontagne. 2017. Virtual reality treatment and assessments for post-stroke unilateral spatial neglect: A systematic literature review. Neuropsychological rehabilitation 27, 3 (2017), 409-454. DOI: 10.1080/09602011.2015.1113187

[6] Vysor, http://www.vysor.io/ 